\title{
Supporting Information: Manipulating Two-Photon-Absorption of Cavity Polaritons by Entangled Light
}

\author{
Bing $\mathrm{Gu}^{*}$ and Shaul Mukame \\ Department of Chemistry and Department of Physics and Astronomy, \\ University of California, Irvine, CA 92697, USA
}

* bingg@uci.edu

† smukamel@uci.edu 


\section{CONTENTS}

\begin{tabular}{ll}
\hline S1. Eq. 4 - Entangled two-photon-absorption & S2
\end{tabular}

S2. Eq. 5 - Sum-over-states expression for the transition amplitude S3

\begin{tabular}{ll}
\hline S3. The twin-photon wavefunction & S4
\end{tabular}

$\begin{array}{ll}\text { S3.A. The correlation amplitude } & \text { S5 }\end{array}$

\begin{tabular}{ll}
\hline 1. Indistinguishable photons & S6
\end{tabular}

$\begin{array}{ll}\text { S3.B. Uncorrelated photons } & \text { S6 }\end{array}$

\begin{tabular}{ll}
\hline S3.C. Coherent states & S7
\end{tabular}

1. Classical light with the same spectral function with quantum light $\quad$ S9

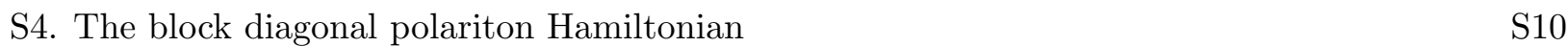

S4.A. Two molecules with different transition frequencies $\quad$ S10

\begin{tabular}{ll}
\hline 1. Single-polariton block & S10
\end{tabular}

$\begin{array}{ll}\text { 2. Two-polariton block } & \text { S10 }\end{array}$

$\begin{array}{ll}\text { S4.B. Two-polariton block for } N \text { identical molecules } & \text { S10 }\end{array}$

$\begin{array}{ll}\text { References } & \text { S12 }\end{array}$

\section{S1. EQ. 4 - ENTANGLED TWO-PHOTON-ABSORPTION}

The two-photon absorption (TPA) signal is defined by the transition probability to a final state $|f\rangle$. In the interaction picture using $H_{0}=H_{\mathrm{p}}+H_{\mathrm{R}}$,

$$
P_{f}(t)=\operatorname{Tr}\left\{|f\rangle\langle f| \rho_{\mathrm{I}}(t)\right\}
$$

where $\rho_{\mathrm{I}}(t)$ is the density matrix in the interaction picture, and where we have taken into account

that $|f\rangle\langle f|$ is time-independent in the interaction picture. The Liouville von-Neumann equation for the joint matter + photon reads

$$
i \frac{\mathrm{d}}{\mathrm{d} t} \rho_{\mathrm{I}}(t)=\left[H_{\mathrm{RM}, \mathrm{I}}(t), \rho_{\mathrm{I}}(t)\right]
$$

The formal solution to Eq. $(\mathrm{S} 2)$ is given by

$$
\rho_{\mathrm{I}}(t)=\mathcal{T} e^{-i \int_{t_{0}}^{t} \mathcal{L}_{\mathrm{RM}, \mathrm{I}}\left(t^{\prime}\right) \mathrm{d} t^{\prime}} \rho_{0}=\mathcal{T} e^{-i \int_{t_{0}}^{t} H_{\mathrm{RM}, \mathrm{I}}\left(t^{\prime}\right) \mathrm{d} t^{\prime}} \rho_{0} \overline{\mathcal{T}} e^{i \int_{t_{0}}^{t} H_{\mathrm{RM}, \mathrm{I}}\left(t^{\prime}\right) \mathrm{d} t^{\prime}} .
$$


where $O_{\mathrm{I}}(t)=e^{+i H_{0}\left(t-t_{0}\right)} O e^{-i H_{0}\left(t-t_{0}\right)}$ and $\mathcal{T}(\overline{\mathcal{T}})$ is the time-ordering (reverse time-ordering) operator. Here $\mathcal{L}_{\mathrm{RM}, \mathrm{I}}(t) \rho=\left[H_{\mathrm{RM}, \mathrm{I}}(t), \rho\right]$ is the Liouvillian superoperator.

Using Eq. (S3) in Eq. (S1) leads to

$$
P_{f}(t)=\operatorname{Tr}\left\{\overline{\mathcal{T}} e^{i \int_{t_{0}}^{t} H_{\mathrm{RM}}(s) \mathrm{d} s}|f\rangle\langle f| \mathcal{T} e^{-i \int_{t_{0}}^{t} H_{\mathrm{RM}}(s) \mathrm{d} s} \rho_{0}\right\},
$$

where we have used the cyclic invariance of the trace $\operatorname{Tr}\{A B\}=\operatorname{Tr}\{B A\}$. To simplify the notation, we have suppressed the subscript I for interaction picture operators, i.e., $O(t) \equiv O_{\mathrm{I}}(t)$. Initially, the system is uncorrelated with the external field, $\rho_{0}=|g\rangle\langle g| \otimes \rho_{\mathrm{R}}(0)$. Expanding the exponentials in Eq. (S4) to second-order in the radiation-matter coupling and retaining the terms leading to two-photon absorption yields

$$
P_{f}(t)=\sum_{\mathbf{p}} \int_{t_{0}}^{t} d t_{2} \int_{t_{0}}^{t_{2}} d t_{1} \int_{t_{0}}^{t} d t_{2}^{\prime} \int_{t_{0}}^{t_{2}^{\prime}} d t_{1}^{\prime}\left\langle g\left|V_{p_{4}}\left(t_{1}^{\prime}\right) V_{p_{3}}\left(t_{2}^{\prime}\right)\right| f\right\rangle\left\langle f\left|V_{p_{2}}^{\dagger}\left(t_{2}\right) V_{p_{1}}^{\dagger}\left(t_{1}\right)\right| g\right\rangle G_{\mathbf{p}}^{(2)}\left(t_{1}^{\prime}, t_{2}^{\prime}, t_{2}, t_{1}\right)
$$

where $G_{\mathbf{p}}^{(2)}\left(t_{1}^{\prime}, t_{2}^{\prime}, t_{2}, t_{1}\right)=\left\langle\hat{E}_{p_{4}}^{\dagger}\left(t_{1}^{\prime}\right) \hat{E}_{p_{3}}^{\dagger}\left(t_{2}^{\prime}\right) \hat{E}_{p_{2}}\left(t_{2}\right) \hat{E}_{p_{1}}\left(t_{1}\right)\right\rangle$ is a field correlation function. Equation (S5) can be represented by the time-loop diagram depicted in Fig. 1c. The subscript $\mathbf{p}=p_{4} p_{3} p_{2} p_{1}$ denotes the sequence of photons $\left(\omega_{1} / \omega_{2}\right)$ that interact with the system along the time-loop clockwise. There are four pathways corresponding to $\mathbf{p}=\{1221,1212,2121,2112\}$, see Fig. 1c. Since $P_{f}(t)=0$ for $f$-states outside the double-excitation manifold, we can sum over all polariton states, which leads to the final compact expression for the ETPA signal

$$
S_{\mathrm{ETPA}}=\sum_{\mathbf{p}} \int_{t_{0}}^{t} d t_{2} \int_{t_{0}}^{t_{2}} d t_{1} \int_{t_{0}}^{t} d t_{2}^{\prime} \int_{t_{0}}^{t_{2}^{\prime}} d t_{1}^{\prime} C_{\mathbf{p}}\left(t_{1}^{\prime}, t_{2}^{\prime}, t_{2}, t_{1}\right) G_{\mathbf{p}}^{(2)}\left(t_{1}^{\prime}, t_{2}^{\prime}, t_{2}, t_{1}\right)
$$

where $C_{\mathbf{p}}\left(t_{1}^{\prime}, t_{2}^{\prime}, t_{2}, t_{1}\right)=\left\langle V_{p_{4}}\left(t_{1}^{\prime}\right) V_{p_{3}}\left(t_{2}^{\prime}\right) V_{p_{2}}^{\dagger}\left(t_{2}\right) V_{p_{1}}^{\dagger}\left(t_{1}\right)\right\rangle$ is the four-point dipole correlation function, and where we have used $I=\sum_{f}|f\rangle\langle f|$.

\section{S2. EQ. 5 - SUM-OVER-STATES EXPRESSION FOR THE TRANSITION AMPLITUDE}

For initially pure two-photon state $\rho_{\mathrm{R}}(0)=|\Phi\rangle\langle\Phi|$, the ETPA signal can be recast as the modulus square of a transition amplitude $S_{\mathrm{ETPA}}=\sum_{f}\left|T_{f g}\right|^{2}$. The transition amplitude for the entangled two-photon absorption reads

$$
T_{f g}=\sum_{p_{1}, p_{2}} \int_{t_{0}}^{t} \mathrm{~d} t_{2} \int_{t_{0}}^{t_{2}} \mathrm{~d} t_{1}\left\langle f\left|V_{p_{2}}^{\dagger}\left(t_{2}\right) V_{p_{1}}^{\dagger}\left(t_{1}\right)\right| g\right\rangle\left\langle 0\left|\hat{E}_{p_{2}}\left(t_{2}\right) \hat{E}_{p_{1}}\left(t_{1}\right)\right| \Phi\right\rangle
$$

Using the many-body eigenstates of the polariton Hamiltonian $\{|i\rangle, i=0,1, \cdots\}$ sorted in ascending order of energy $\left\{\omega_{i}\right\}$, the raising dipole operator $V_{n}^{\dagger}$ can be written as [1]

$$
V_{n}^{\dagger}(t)=-\sum_{i<j} e^{i \omega_{j i} t} \boldsymbol{\mu}_{j i} \cdot \mathbf{e}_{n}|j\rangle\langle i| .
$$


where $\boldsymbol{\mu}_{j i}=\langle j|\boldsymbol{\mu}| i\rangle$ is the dipole matrix element. Inserting Eq. (S8) into Eq. (S7) yields Eq. (5) in the main text

$$
T_{f g}(t)=\sum_{p_{1} \neq p_{2}} \sum_{e} D_{p_{2} p_{1}}^{(e)} \int_{t_{0}}^{t} \mathrm{~d} t_{2} e^{i \omega_{f e} t_{2}} \int_{t_{0}}^{t_{2}} \mathrm{~d} t_{1} e^{i \omega_{e g} t_{1}} \Phi_{p_{2} p_{1}}\left(t_{2}, t_{1}\right)
$$

where $\Phi_{p_{2} p_{1}}\left(t_{2}, t_{1}\right)=\left\langle 0\left|\hat{E}_{p_{2}}\left(t_{2}\right) \hat{E}_{p_{1}}\left(t_{1}\right)\right| \Phi\right\rangle$ is proportional to the amplitude of detecting photon $p_{1}$ at $t_{1}$ and photon $p_{2}$ at $t_{2}$. Intuitively, the matter can be taken as a photon detector with a complex inner structure.

It is useful to have the frequency-domain expression for the transition amplitude. The twophoton amplitude in the time domain can be written as

$$
\Phi_{p_{2} p_{1}}\left(t_{2}, t_{1}\right)=\iint \mathrm{d} \omega_{2} \mathrm{~d} \omega_{1} e^{-i \omega_{p_{1}} t_{1}} e^{-i \omega_{p_{2}} t_{2}} \Phi_{p_{2} p_{1}}\left(\omega_{p_{2}}, \omega_{p_{1}}\right)
$$

where $\Phi_{p_{2} p_{1}}\left(\omega_{2}, \omega_{1}\right) \equiv\left\langle 0\left|\hat{E}_{p_{2}}\left(\omega_{2}\right) \hat{E}_{p_{1}}\left(\omega_{1}\right)\right| \Phi\right\rangle, \hat{E}_{j}(t)=\int \mathrm{d} \omega \hat{E}_{j}(\omega) e^{-i \omega t}$, and $\hat{E}_{j}(\omega)=i \sqrt{\frac{\omega}{2 \epsilon_{0} \mathcal{V}}} b_{j}(\omega)$. Inserting Eq. (S10) into Eq. (S9) leads to the frequency-domain expression for the transition amplitude (up to a global phase)

$T_{f g}=\sum_{e} \iint \mathrm{d} \omega_{1} \mathrm{~d} \omega_{2} \frac{1}{\omega_{f g}-i \gamma_{f}-\omega_{1}-\omega_{2}}\left(D_{12}^{(e)} \frac{\left\langle 0\left|\hat{E}_{1}\left(\omega_{1}\right) \hat{E}_{2}\left(\omega_{2}\right)\right| \Phi\right\rangle}{\omega_{e g}-\omega_{2}-i \gamma_{e}}+D_{21}^{(e)} \frac{\left\langle 0\left|\hat{E}_{2}\left(\omega_{2}\right) \hat{E}_{1}\left(\omega_{1}\right)\right| \Phi\right\rangle}{\omega_{e g}-\omega_{1}-i \gamma_{e}}\right)$

\section{S3. THE TWIN-PHOTON WAVEFUNCTION}

We consider the following twin-photon wavefunction produced by parametric down conversion (PDC) [2, 3]

$$
\phi\left(\omega_{1}, \omega_{2}\right)=\mathcal{N} A\left(\omega_{1}+\omega_{2}\right) \operatorname{sinc}(\Delta k L / 2)
$$

where $L$ is the crystal length, $\Delta k=k_{\mathrm{p}}-\left(k_{1}+k_{2}\right), A\left(\omega_{1}+\omega_{2}\right)=\left(\frac{1}{\sigma \sqrt{\pi}}\right)^{1 / 2} \exp \left(-\frac{\left(\omega_{1}+\omega_{2}-\omega_{\mathrm{p}}\right)^{2}}{\sigma^{2}}\right)$ is the normalized pump pulse envelope with bandwidth $\sigma, \operatorname{sinc}(x)=\sin (x) / x, \mathcal{N}$ the normalization constant. For a narrow-band pump $\sigma \rightarrow 0$, the spectral envelope

$$
A\left(\omega_{1}+\omega_{2}\right) \approx \delta\left(\omega_{1}+\omega_{2}-\omega_{\mathrm{p}}\right)
$$

which reflects energy conservation in the PDC process. In type-II down-conversion where $\mathbf{e}_{1}$ and $\mathbf{e}_{2}$ are orthogonal, we can expand the wave vector in a Taylor series around the central frequency

$$
k_{j}\left(\Delta \omega_{j}+\omega_{j}^{0}\right) \approx k_{j}\left(\omega_{j}^{0}\right)+v_{j}^{-1} \Delta \omega_{j},
$$


and the phase-matching condition may be approximated to linear order as

$$
\Delta k L / 2=\frac{1}{2}\left(\Delta \omega_{1} T_{1}+\Delta \omega_{2} T_{2}\right)
$$

where $\Delta \omega_{j}=\omega_{j}-\omega_{j}^{0}$, and the transit time difference $T_{j}=L / v_{\mathrm{p}}-L / v_{j}$ for $j=1,2$ with $v_{j}=$ $\nabla_{k_{j}} \omega_{j}\left(k_{j}^{0}\right)$ is the group velocity. With Eqs. S15) and (S13), The PDC two-photon wavefunction generated from a monochromatic pump becomes Eq. (7)

$$
\phi\left(\omega_{1}, \omega_{2}\right)=\mathcal{N} \delta\left(\omega_{1}+\omega_{2}-\omega_{\mathrm{p}}\right) \operatorname{sinc}\left(\frac{\Delta \omega_{1} T}{2}\right)
$$

where $T=T_{1}-T_{2}$ is the entanglement time characterising the arrival time delay between the two photons.

In the time domain, the two-photon wavefunction reads

$$
\phi\left(t_{1}, t_{2}\right) \equiv \int \mathrm{d} \omega_{1} \int \mathrm{d} \omega_{2} e^{-i \omega_{1} t_{1}-i \omega_{2} t_{2}} \phi\left(\omega_{1}, \omega_{2}\right)=\frac{2 \pi \mathcal{N}}{T} e^{-i \omega_{1}^{0} t_{1}-i \omega_{2}^{0} t_{2}} \Pi\left(\frac{t_{1}-t_{2}}{T}\right)
$$

where the rectangular function $\Pi(x)=1$ for $-\frac{1}{2}<x<\frac{1}{2}$ and 0 otherwise and $\omega_{i}^{0}$ is the central frequency of the $i$-th beam. Equation (S17) reflects the time-correlation between the entangled photons: the arrival time of each photon is random, but they must arrive together within the entanglement time. Note that the two photons are not time-ordered $\left(\omega_{1}\right.$ can come before or after $\left.\omega_{2}\right)$ as in the quantum light generated by atomic cascade [4].

\section{S3.A. The correlation amplitude}

Here we establish the connection between the two-photon correlation amplitude $\Phi$ and the two-photon wavefunction $\phi$. The two-photon correlation amplitude can be obtained by

$$
\begin{aligned}
\Phi_{21}\left(\omega_{2}, \omega_{1}\right) & =\iint \mathrm{d} \omega_{1}^{\prime} \mathrm{d} \omega_{2}^{\prime} \phi\left(\omega_{1}^{\prime}, \omega_{2}^{\prime}\right)\left\langle 0\left|\hat{E}_{2}\left(\omega_{2}\right) \hat{E}_{1}\left(\omega_{1}\right) a_{1}^{\dagger}\left(\omega_{1}^{\prime}\right) a_{2}^{\dagger}\left(\omega_{2}^{\prime}\right)\right| 0\right\rangle \\
& =-\frac{\sqrt{\omega_{2} \omega_{1}}}{2 \epsilon_{0} \mathcal{V}} \iint \mathrm{d} \omega_{1}^{\prime} \mathrm{d} \omega_{2}^{\prime} \phi\left(\omega_{1}^{\prime}, \omega_{2}^{\prime}\right)\left\langle 0\left|a_{2}\left(\omega_{2}\right) a_{1}\left(\omega_{1}\right) a_{1}^{\dagger}\left(\omega_{1}^{\prime}\right) a_{2}^{\dagger}\left(\omega_{2}^{\prime}\right)\right| 0\right\rangle
\end{aligned}
$$

For distinguishable photons,

$$
\left\langle 0\left|a_{2}\left(\omega_{2}\right) a_{1}\left(\omega_{1}\right) a_{1}^{\dagger}\left(\omega_{1}^{\prime}\right) a_{2}^{\dagger}\left(\omega_{2}^{\prime}\right)\right| 0\right\rangle=\delta\left(\omega_{1}-\omega_{1}^{\prime}\right) \delta\left(\omega_{2}-\omega_{2}^{\prime}\right) .
$$

Then

$$
\Phi_{21}\left(\omega_{2}, \omega_{1}\right)=-\frac{\sqrt{\omega_{2} \omega_{1}}}{2 \epsilon_{0} \mathcal{V}} \phi\left(\omega_{1}, \omega_{2}\right)
$$

and in the time-domain

$$
\Phi_{21}\left(t_{2}, t_{1}\right) \approx-\frac{\sqrt{\omega_{2}^{0} \omega_{1}^{0}}}{2 \epsilon_{0} \mathcal{V}} \phi\left(t_{1}, t_{2}\right)
$$


where we have invoked the slowly varying approximation

$$
\frac{\sqrt{\omega_{2} \omega_{1}}}{2 \epsilon_{0} \mathcal{V}} \approx \frac{\sqrt{\omega_{2}^{0} \omega_{1}^{0}}}{2 \epsilon_{0} \mathcal{V}}
$$

Similarly,

$$
\Phi_{12}\left(\omega_{2}, \omega_{1}\right)=-\frac{\sqrt{\omega_{2} \omega_{1}}}{2 \epsilon_{0} \mathcal{V}} \phi\left(\omega_{2}, \omega_{1}\right)
$$

and

$$
\Phi_{12}\left(t_{2}, t_{1}\right)=\iint \mathrm{d} \omega_{2} \mathrm{~d} \omega_{1} e^{-i \omega_{2} t_{1}} e^{-i \omega_{1} t_{2}} \Phi_{12}\left(\omega_{1}, \omega_{2}\right) \approx-\frac{\sqrt{\omega_{2}^{0} \omega_{1}^{0}}}{2 \epsilon_{0} \mathcal{V}} \phi\left(t_{2}, t_{1}\right)
$$

These relations allow us to obtain the correlation amplitude from the two-photon wavefunction.

\section{Indistinguishable photons}

The distinguishing characteristics by polarization or arrival time in the entangled photons can be eliminated such that they become indistinguishable [5].

For indistinguishable photons, we can suppress the photon index such that

$$
\left\langle 0\left|a\left(\omega_{2}\right) a\left(\omega_{1}\right) a^{\dagger}\left(\omega_{1}^{\prime}\right) a^{\dagger}\left(\omega_{2}^{\prime}\right)\right| 0\right\rangle=\delta\left(\omega_{1}-\omega_{1}^{\prime}\right) \delta\left(\omega_{2}-\omega_{2}^{\prime}\right)+\delta\left(\omega_{1}-\omega_{2}^{\prime}\right) \delta\left(\omega_{2}-\omega_{1}^{\prime}\right)
$$

Then

$$
\Phi_{21}\left(\omega_{2}, \omega_{1}\right)=\Phi_{12}\left(\omega_{2}, \omega_{1}\right)=-\frac{\sqrt{\omega_{2} \omega_{1}}}{2 \epsilon_{0} \mathcal{V}}\left(\phi\left(\omega_{1}, \omega_{2}\right)+\phi\left(\omega_{2}, \omega_{1}\right)\right)
$$

It follows that $\Phi_{12}\left(t_{2}, t_{1}\right)=\Phi_{21}\left(t_{2}, t_{1}\right)$ meaning that the transition amplitudes associated with pathways involving the same intermediate state but a different photon sequences coincide. This implies that matter cannot distinguish the interacting photons.

\section{S3.B. Uncorrelated photons}

For uncorrelated single photons, the two-photon wavefunction can be factorized as

$$
\phi\left(\omega_{1}, \omega_{2}\right)=\phi_{1}\left(\omega_{1}\right) \phi_{2}\left(\omega_{2}\right)
$$

and so is the detection amplitude $\tilde{\Phi}_{p_{2} p_{1}}\left(t, t^{\prime}\right) \equiv\left\langle 0\left|a_{p_{2}}(t) a_{p_{1}}\left(t^{\prime}\right)\right| \Phi\right\rangle$ representing the probability ampliutude of observing photon $p_{1}$ at time $t^{\prime}$ and photon $p_{2}$ at time $t$,

$$
\tilde{\Phi}_{p_{2} p_{1}}\left(t, t^{\prime}\right) \equiv\left\langle 0\left|a_{p_{2}}(t)\right| \phi_{p_{2}}\right\rangle\left\langle 0\left|a_{p_{1}}\left(t^{\prime}\right)\right| \phi_{p_{1}}\right\rangle
$$


The detection amplitude is proportional to the correlation amplitude under the approximation in Eq. S22). The single-photon state reads

$$
\left|\phi_{j}\right\rangle=\int \mathrm{d} \omega_{j} \phi_{j}\left(\omega_{j}\right) b_{j}^{\dagger}\left(\omega_{j}\right)|0\rangle
$$

where the vaccum corresponds to the modes associated with $j$-th photon. Inserting Eq. (S29) into Eq. (S28) leads to

$$
\begin{aligned}
\tilde{\Phi}_{12}\left(t_{1}, t_{2}\right) & =\left\langle 0\left|b_{1}\left(t_{1}\right)\right| \varphi\right\rangle\left\langle 0\left|b_{2}\left(t_{2}\right)\right| \chi\right\rangle \\
& =\int \mathrm{d} \omega \int \mathrm{d} \omega_{1} e^{-i \omega t_{1}} \varphi\left(\omega_{1}\right)\left\langle 0\left|b_{1}(\omega) a_{1}^{\dagger}\left(\omega_{1}\right)\right| 0\right\rangle \int \mathrm{d} \omega^{\prime} \int \mathrm{d} \omega_{2} \chi\left(\omega_{2}\right) e^{-i \omega^{\prime} t_{2}}\left\langle 0\left|b_{2}\left(\omega^{\prime}\right) a_{2}^{\dagger}\left(\omega_{2}\right)\right| 0\right\rangle \\
& =\phi_{1}\left(t_{1}\right) \phi_{2}\left(t_{2}\right)
\end{aligned}
$$

where $\phi(t)=\int \mathrm{d} \omega e^{-i \omega t} \phi(\omega)$. Similarly,

$$
\tilde{\Phi}_{21}\left(t_{1}, t_{2}\right)=\phi_{1}\left(t_{2}\right) \phi_{2}\left(t_{1}\right)
$$

Insering Eqs. (S30) and S31) into Eq. S9 leads to the transition amplitude for uncorrelated photons

$$
T_{f g}=\frac{\sqrt{\omega_{1}^{0} \omega_{2}^{0}}}{2 \mathcal{V} \epsilon_{0}} \sum_{e} \iint \mathrm{d} \omega_{1} \mathrm{~d} \omega_{2} \frac{\phi_{1}\left(\omega_{1}\right) \phi_{2}\left(\omega_{2}\right)}{\omega_{1}+\omega_{2}-\omega_{f g}+i \gamma_{f}}\left(\frac{D_{12}^{(e)}}{\omega_{e g}-\omega_{2}-i \gamma_{e}}+\frac{D_{21}^{(e)}}{\omega_{e g}-\omega_{1}-i \gamma_{e}}\right)
$$

If the two photons are narrowband with central frequencies $\omega_{j}^{0}$ such that

$$
\phi\left(\omega_{1}\right) \approx \phi\left(\omega_{1}^{0}\right) \delta\left(\omega_{1}-\omega_{1}^{0}\right)
$$

the transition amplitude reduces to

$$
T_{f g}=\frac{\sqrt{\omega_{1}^{0} \omega_{2}^{0}}}{2 \mathcal{V} \epsilon_{0}} \frac{1}{\omega_{1}^{0}+\omega_{2}^{0}-\omega_{f g}+i \gamma_{f}} \phi_{1}\left(\omega_{1}^{0}\right) \phi_{2}\left(\omega_{2}^{0}\right) \sum_{e}\left(D_{12}^{(e)} \frac{1}{\Delta_{e}^{(2)}-i \gamma_{e}}+D_{21}^{(e)} \frac{1}{\Delta_{e}^{(1)}-i \gamma_{e}}\right)
$$

\section{S3.C. Coherent states}

If the photons are in coherent states corresponding to the semiclassical light, the detection amplitude is given by

$$
\phi_{p_{2} p_{1}}\left(t, t^{\prime}\right)=\left\langle\phi_{p_{2}}\left|a_{p_{2}}(t)\right| \phi_{p_{2}}\right\rangle\left\langle\phi_{p_{1}}\left|a_{p_{1}}\left(t^{\prime}\right)\right| \phi_{p_{1}}\right\rangle
$$

The difference between the coherent state and the single-photon state is that annihilation of an photon does not change the photon state in the former whereas it projects the photon state to the vacuum for the latter. 
Coherent states can be generally defined as

$$
\left|\phi_{j}\right\rangle=e^{\alpha A_{j}^{\dagger}-\alpha_{j}^{*} A_{j}}|0\rangle
$$

where $A_{j}^{\dagger} \equiv \int_{0}^{\infty} \mathrm{d} \omega \mathcal{A}(\omega) b_{j}^{\dagger}(\omega)$ is a single-photon creation operator. For a single photon mode,

$$
A_{j}(t)=b_{j}(\omega)
$$

This corresponds to a monochromatic light. For a continuum of modes,

$$
A_{j}^{\dagger}=\int \mathrm{d} \omega \phi_{j}(\omega) b_{j}^{\dagger}(\omega)
$$

where $\phi_{j}(\omega)$ is the normalized spectral envelope. It follows that Eq. S35 becomes

$$
\phi_{21}\left(t, t^{\prime}\right)=\int \mathrm{d} \omega_{2} e^{-i \omega_{2} t} \alpha_{2} \phi_{2}\left(\omega_{2}\right) \int \mathrm{d} \omega_{1} e^{-i \omega_{1} t^{\prime}} \alpha_{1} \phi_{1}\left(\omega_{1}\right)
$$

Realizing that the expectation value of the electric field operator is given by

$$
E_{j}(\omega)=i \sqrt{\frac{\hbar \omega}{2 \epsilon_{0} \mathcal{V}}} \alpha_{j} \phi_{j}(\omega)
$$

Eq. (S39) becomes

$$
\Phi_{21}\left(t, t^{\prime}\right)=\int \mathrm{d} \omega_{2} e^{-i \omega_{2} t} E_{2}\left(\omega_{2}\right) \int \mathrm{d} \omega_{1} e^{-i \omega_{1} t^{\prime}} E_{1}\left(\omega_{1}\right)=E_{2}(t) E_{1}\left(t^{\prime}\right)
$$

Equation (S41) implies that the detection amplitude is simply the product of the electric fields, consistent with a semiclassical picture of photon detection theory [6].

The two-photon transition amplitude can be then obtained by inserting Eq. (S41) into Eq. (S34)

$$
T_{f g}(t)=\sum_{p_{1} \neq p_{2}} \sum_{e} D_{p_{2} p_{1}}^{(e)} \int_{t_{0}}^{t} \mathrm{~d} t_{2} e^{i \omega_{f e} t_{2}} E_{p_{2}}\left(t_{2}\right) \int_{t_{0}}^{t_{2}} \mathrm{~d} t_{1} e^{i \omega_{e g} t_{1}} E_{p_{1}}\left(t_{1}\right)
$$

The corresponding frequency-domain expression reads

$$
T_{f g}=\sum_{e} \iint \mathrm{d} \omega_{1} \mathrm{~d} \omega_{2} \frac{E_{1}\left(\omega_{1}\right) E_{2}\left(\omega_{2}\right)}{\omega_{f g}-i \gamma_{f}-\omega_{1}-\omega_{2}}\left(\frac{D_{12}^{(e)}}{\omega_{e g}-\omega_{2}-i \gamma_{e}}+\frac{D_{21}^{(e)}}{\omega_{e g}-\omega_{1}-i \gamma_{e}}\right) .
$$

Thus, we have obtained the classical two-photon absorption amplitude from a fully quantum mechanical treatment. For monochromatic fields $E_{j}\left(\omega_{j}\right)=E_{j} \delta\left(\omega_{j}-\omega_{j}^{0}\right)$, Eq. S43 reduces to

$$
T_{f g}=\sum_{e} \frac{E_{1}\left(\omega_{1}^{0}\right) E_{2}\left(\omega_{2}^{0}\right)}{\omega_{f g}-i \gamma_{f}-\omega_{1}^{0}-\omega_{2}^{0}}\left(\frac{D_{12}^{(e)}}{\Delta_{e}^{(2)}-i \gamma_{e}}+\frac{D_{21}^{(e)}}{\Delta_{e}^{(1)}-i \gamma_{e}}\right)
$$


TABLE S1. Expressions for two-photon-absorption signal with quantum and classical light. The photon indexes $p_{2} p_{1}=\{21,12\}$ depending on which photon interacts with the matter first.

\section{Time domain}

\begin{tabular}{ll}
\hline classical light & $T_{f g}(t)=\sum_{p_{1} \neq p_{2}} \sum_{e} D_{p_{2} p_{1}}^{(e)} \int_{t_{0}}^{t} \mathrm{~d} t_{2} e^{i \omega_{f e} t_{2}} E_{p_{2}}\left(t_{2}\right) \int_{t_{0}}^{t_{2}} \mathrm{~d} t_{1} e^{i \omega_{e g} t_{1}} E_{p_{1}}\left(t_{1}\right)$ \\
quantum light & $T_{f g}(t)=\sum_{p_{1} \neq p_{2}} \sum_{e} D_{p_{2} p_{1}}^{(e)} \int_{t_{0}}^{t} \mathrm{~d} t_{2} e^{i \omega_{f e} t_{2}} \int_{t_{0}}^{t_{2}} \mathrm{~d} t_{1} e^{i \omega_{e g} t_{1}} \Phi_{p_{2} p_{1}}\left(t_{2}, t_{1}\right)$ \\
\hline
\end{tabular}

Frequency domain

classical light $\quad T_{f g}=-\sum_{p_{1} \neq p_{2}} \sum_{e} \iint \mathrm{d} \omega_{1} \mathrm{~d} \omega_{2} \frac{E_{1}\left(\omega_{1}\right) E_{2}\left(\omega_{2}\right)}{\omega_{f g}-i \gamma_{f}-\omega_{1}-\omega_{2}}\left(\frac{D_{p_{2} p_{1}}^{(e)}}{\omega_{e g}-\omega_{p_{1}}-i \gamma_{e}}\right)$

quantum light $T_{f g}=-\sum_{e} \sum_{p_{2} \neq p_{1}} \iint \mathrm{d} \omega_{1} \mathrm{~d} \omega_{2} \frac{1}{\omega_{f g}-i \gamma_{f}-\omega_{1}-\omega_{2}}\left(D_{p_{2} p_{1}}^{(e)} \frac{\left\langle 0\left|E_{p_{2}}\left(\omega_{p_{2}}\right) E_{p_{1}}\left(\omega_{p_{1}}\right)\right| \Phi\right\rangle}{\omega_{e g}-\omega_{p_{1}}-i \gamma_{e}}\right)$

\section{Classical light with the same spectral function with quantum light}

If the classical light have the same spectral function as in the quantum light,

$$
E_{j}\left(\omega_{j}\right)=E_{j}^{0} \frac{T}{2 \pi} \operatorname{sinc}\left(\Delta \omega_{j} T / 2\right)
$$

Using the identity $\int_{-\infty}^{+\infty} \operatorname{sinc}(\omega T / 2) e^{-i \omega t} \mathrm{~d} \omega=\frac{2 \pi}{T} \Pi\left(\frac{t}{T}\right)$, the pulse envelope reads

$$
E_{j}(t)=E_{j}^{0} e^{-i \omega_{j}^{0} t} \Pi\left(\frac{t}{T}\right)
$$

Inserting Eq. (S46) into Eq. S42 leads to the two-photon transition amplitude with two rectangular pulses $\left(t_{0} \rightarrow-\infty, t \rightarrow \infty\right)$

$$
\begin{aligned}
T_{f g} & =E_{2}^{0} E_{1}^{0} \sum_{p_{1} \neq p_{2}} \sum_{e} D_{p_{2} p_{1}}^{(e)} \int_{-\infty}^{\infty} \mathrm{d} t_{2} e^{i\left(\omega_{f e}-\omega_{p_{2}}^{0}\right) t_{2}} \Pi\left(\frac{t_{2}}{T}\right) \int_{-\infty}^{t_{2}} \mathrm{~d} t_{1} e^{i\left(\omega_{e g}-\omega_{p_{1}}^{0}\right) t_{1}} \Pi\left(\frac{t_{1}}{T}\right) \\
& =E_{2}^{0} E_{1}^{0} \sum_{p_{1} \neq p_{2}} \sum_{e} D_{p_{2} p_{1}}^{(e)} \int_{-T / 2}^{T / 2} \mathrm{~d} t_{2} e^{i\left(\omega_{f e}-\omega_{p_{2}}^{0}\right) t_{2}} \frac{1}{i \Delta_{e}^{\left(p_{1}\right)}}\left(e^{i \Delta_{e}^{\left(p_{1}\right)} t_{2}}-e^{-i \Delta_{e}^{\left(p_{1}\right)} T / 2}\right) \\
& =-E_{2}^{0} E_{1}^{0} \sum_{p_{1} \neq p_{2}} \sum_{e} D_{p_{2} p_{1}}^{(e)}\left(\frac{2 i \operatorname{sinc}\left(\left(\omega_{f g}-\omega_{1}^{0}-\omega_{2}^{0}\right) T / 2\right)}{\omega_{e g}-\omega_{p_{1}}^{0}}+\frac{e^{-i\left(\omega_{e g}-\omega_{p_{1}}^{0}\right) T / 2} 2 i \operatorname{sinc}\left(\left(\omega_{f e}-\omega_{p_{2}}^{0}\right) T / 2\right)}{\omega_{e g}-\omega_{p_{1}}^{0}}\right)
\end{aligned}
$$

We have assumed that the pulse duration is shorter than the lifetime, i.e., $T \ll \gamma_{e}^{-1}$. The first term contains the two-photon resonance condition and thus represents the TPA process whereas the second term contains two single-photon resonances representing a sequential excitation. As shown, for the uncorrelated light, varying the spectral width $1 / T$ does not allow modifying the transition amplitude for each transition pathways. 


\section{S4. THE BLOCK DIAGONAL POLARITON HAMILTONIAN}

\section{S4.A. Two molecules with different transition frequencies}

\section{Single-polariton block}

For $N=2$ molecules, the subspace Hamiltonian in single-polariton subspace reads

$$
H^{(1)}=\left[\begin{array}{ccc}
\omega_{\mathrm{c}} & g_{0} & g_{0} \\
g_{0} & \omega_{\mathrm{a}} & 0 \\
g_{0} & 0 & \omega_{\mathrm{b}}
\end{array}\right]
$$

where $g_{0}$ is the single-molecule coupling strength.

\section{Two-polariton block}

The two-polariton block Hamiltonian spanned by the basis $|g g 2\rangle,|e g 1\rangle,|g e 1\rangle,|e e 0\rangle$ reads

$$
H^{(2)}=\left[\begin{array}{cccc}
2 \omega_{\mathrm{c}} & \sqrt{2} g_{0} & \sqrt{2} g_{0} & 0 \\
\sqrt{2} g_{0} & \omega_{\mathrm{a}}+\omega_{\mathrm{c}} & 0 & g_{0} \\
\sqrt{2} g_{0} & 0 & \omega_{\mathrm{b}}+\omega_{\mathrm{c}} & g_{0} \\
0 & g_{0} & g_{0} & \omega_{\mathrm{a}}+\omega_{\mathrm{b}}
\end{array}\right]
$$

Solving $\operatorname{det}\left(\omega-H^{(2)}\right)=0$ yields the polariton energies $\omega=2 \omega_{\mathrm{c}} \pm \sqrt{\delta^{2}+6 g_{0}^{2}}, 2 \omega_{\mathrm{c}}$.

\section{S4.B. Two-polariton block for $N$ identical molecules}

We now consider $N$ identical molecules with transition frequency $\omega_{0}$ and coupling $g_{j}=g_{0}$. To understand the structure of the two-polariton states, it is convenient to introduce the collective exciton operators

$$
X_{j}^{\dagger}=\frac{1}{\sqrt{N}} \sum_{n=1}^{N} e^{i k_{j} n} \sigma_{n}^{\dagger}, \quad j=0,1 \cdots, N-1,
$$

where $k_{j}=2 \pi j / N, j=0,1, \cdots, N-1$. The collective exciton operators satisfy the commutation relations

$$
\left[X_{i}, X_{j}^{\dagger}\right]=-\frac{1}{N} \sum_{n} e^{i\left(k_{i}-k_{j}\right) n} \sigma_{n}^{z}=\delta_{i j}-\frac{2}{N} \sum_{n} e^{i\left(k_{i}-k_{j}\right) n} \sigma_{n}^{\dagger} \sigma_{n}
$$


Since these are different from the boson commutation relations, the excitons cannot in general be considered as bosons. In the low excitation limit of many molecules, i.e., $\sum_{n=1}^{N} \sigma_{n}^{\dagger} \sigma_{n} \ll N$, Eq. (S51) becomes

$$
\left[X_{i}, X_{j}^{\dagger}\right]=\delta_{i j}+\mathcal{O}\left(N^{-1}\right)
$$

and the collective excitons are approximately bosons.

The upper and lower polaritons are admixtures of the bright exciton state $\left|\mathrm{X}_{0}\right\rangle=X_{0}^{\dagger}|G\rangle$, where $|G\rangle$ is the ground state for all molecules, and the cavity mode, with an enhanced splitting $2 g_{0} \sqrt{N}$. The double-excitation manifold contains $\frac{N(N+1)}{2}+1$ states. The polariton Hamiltonian can be recasted in terms of the collective exciton operators

$$
H_{\mathrm{p}}=\omega_{0} \sum_{j=0}^{N-1} X_{j}^{\dagger} X_{j}+\omega_{\mathrm{c}} a^{\dagger} a+g_{0} \sqrt{N}\left(X_{0}^{\dagger} a+X_{0} a^{\dagger}\right) .
$$

The double-excitation space can be decomposed into three subspaces spanned, respectively, by

$$
\left\{|2\rangle,\left|\mathrm{X}_{0} 1\right\rangle,\left|\mathrm{X}_{0} \mathrm{X}_{0}\right\rangle=\sqrt{\frac{N-1}{N}}\left(X_{0}^{\dagger}\right)^{2}|g\rangle\right\}
$$

$\left\{\left|\mathrm{X}_{j} \mathrm{X}_{0}\right\rangle,\left|\mathrm{X}_{j} 1\right\rangle, j \neq 0\right\}$, and $\left\{\left|\mathrm{X}_{j} \mathrm{X}_{k}\right\rangle, j, k \neq 0\right\}$. The first block comes from excitations of bright excitons and cavity photons and contains three two-polariton states. The subblock Hamiltonian reads

$$
H=\left[\begin{array}{ccc}
2 \omega_{c} & g_{0} \sqrt{2 N} & 0 \\
g_{0} \sqrt{2 N} & \omega_{0}+\omega_{\mathrm{c}} & g_{0} N / \sqrt{N-1} \\
0 & g_{0} N / \sqrt{N-1} & 2 \omega_{0}
\end{array}\right]
$$

Eigenvalues of this Hamiltonian Eq. (S55) leads to a pair of upper and lower two-polaritons and one middle two-polariton

$$
\left|f_{\mathrm{M}}\right\rangle=\left[\sqrt{\frac{2(N-1)}{3 N-2}}, 0, \sqrt{\frac{N}{3 N-2}}\right]
$$

at $2 \omega_{\mathrm{c}}$. The enhanced coupling between $|2\rangle$ and $\left|\mathrm{X}_{0} 1\right\rangle$ due to the presence of cavity photons is responsible for the enhanced upper and lower two-polariton splitting.

In addition to this splitting, there is another polariton pair from the second subspace involving dark exciton excitation and cavity photons. The Hamiltonian in this subblock spanned by states $\left|X_{j} 1\right\rangle$ and $\left|X_{j} X_{0}\right\rangle$ is given by (for each $j$ )

$$
H=\left[\begin{array}{cc}
\omega_{0}+\omega_{\mathrm{c}} & g \sqrt{\frac{N-2}{N}} \\
g \sqrt{\frac{N-2}{N}} & 2 \omega_{0}
\end{array}\right]
$$


where $g=g_{0} \sqrt{N}$. The splitting is slightly reduced compared to the vacuum Rabi splitting by a factor of $\sqrt{\frac{N-2}{N}}$. Such states are not dipole connected to the upper and lower polaritons, and cannot be observed in the TPA. The third block involves only dark exciton excitations.

[1] Konstantin E. Dorfman, Frank Schlawin, and Shaul Mukamel, "Nonlinear optical signals and spectroscopy with quantum light," Rev. Mod. Phys. 88 (2016).

[2] C. K. Hong and L. Mandel, "Theory of parametric frequency down conversion of light," Phys. Rev. A 31, 2409-2418 (1985).

[3] Timothy E. Keller and Morton H. Rubin, "Theory of two-photon entanglement for spontaneous parametric down-conversion driven by a narrow pump pulse," Phys. Rev. A 56, 1534-1541 (1997).

[4] Ashok Muthukrishnan, Girish S. Agarwal, and Marlan O. Scully, "Inducing Disallowed Two-Atom Transitions with Temporally Entangled Photons," Phys. Rev. Lett. 93, 093002 (2004).

[5] W. P. Grice, A. B. U'Ren, and I. A. Walmsley, "Eliminating frequency and space-time correlations in multiphoton states," Phys. Rev. A 64, 063815 (2001).

[6] Roy J. Glauber, "The Quantum Theory of Optical Coherence," Phys. Rev. 130, 2529-2539 (1963). 